\title{
Time-dependent probability density function in cubic stochastic processes
}

\author{
Eun-jin Kim \\ School of Mathematics and Statistics, University of Sheffield, Sheffield, S3 7RH, United Kingdom \\ Rainer Hollerbach \\ Department of Applied Mathematics, University of Leeds, Leeds, LS2 9JT, United Kingdom \\ (Received 28 June 2016; revised manuscript received 23 August 2016; published 10 November 2016)
}

\begin{abstract}
We report time-dependent probability density functions (PDFs) for a nonlinear stochastic process with a cubic force using analytical and computational studies. Analytically, a transition probability is formulated by using a path integral and is computed by the saddle-point solution (instanton method) and a new nonlinear transformation of time. The predicted PDF $p(x, t)$ in general involves a time integral, and useful PDFs with explicit dependence on $x$ and $t$ are presented in certain limits (e.g., in the short and long time limits). Numerical simulations of the Fokker-Planck equation provide exact time evolution of the PDFs and confirm analytical predictions in the limit of weak noise. In particular, we show that transient PDFs behave drastically differently from the stationary PDFs in regard to the asymmetry (skewness) and kurtosis. Specifically, while stationary PDFs are symmetric with the kurtosis smaller than 3, transient PDFs are skewed with the kurtosis larger than 3; transient PDFs are much broader than stationary PDFs. We elucidate the effect of nonlinear interaction on the strong fluctuations and intermittency in the relaxation process.
\end{abstract}

DOI: 10.1103/PhysRevE.94.052118

\section{INTRODUCTION}

Many systems in nature or laboratories not only involve stochastic processes due to intrinsic variability, or to uncertainty in the system, but may also be far from equilibrium. Computing the time evolution of the probability density function (PDF) of such systems is often a major challenge. While in thermal equilibrium the same level of fluctuations can be maintained by a reservoir (e.g., heat bath) at a fixed temperature (e.g., by the fluctuation-dissipation theorem [1]), far from equilibrium such a reservoir no longer exists. The level of fluctuations in the system thus changes with time and becomes a dynamical variable itself. For instance, drastic change in fluctuations can be caused by a sudden change in the temperature at the reservoir, or just by an initial out-of-equilibrium PDF. Consequently, a full knowledge of the time evolution of PDFs becomes critical.

As a simplest model, a Gaussian process has been widely used to understand a variety of stochastic processes [2,3]. At the heart of a Gaussian process is Brownian motion (random walk) driven by white noise (random noise with a very short memory), where mean square displacement increases linearly with time while there is no change in mean displacement. When subject to a linear force, it becomes the OrnsteinUhlenbeck $(\mathrm{O}-\mathrm{U})$ process, which is a prototypical model for a noisy relaxation system, heavily used and extended in many areas of physical science and financial mathematics (e.g., Refs. [2,3]). This model is governed by the following Langevin equation for a random variable $x$ (say, the position):

$$
\frac{d x}{d t}=-\frac{\partial V}{\partial x}+\xi
$$

where a linear force $\frac{\partial V}{\partial x}=\mu_{o} x$ is given by a quadratic potential $V(x)=\mu_{o} x^{2} / 2$. Here $\mu_{o}$ is a non-negative constant and $\xi$ is the white noise characterized by the following statistical property:

$$
\left\langle\xi(t) \xi\left(t^{\prime}\right)\right\rangle=D \delta\left(t-t^{\prime}\right),
$$

where $D$ is a constant. The angular brackets in Eq. (2) represent the ensemble average over the noise. Given the initial condition (initial PDF), the time evolution of the PDF in the subsequent time is precisely known as the joint distribution with a Gaussian transition probability. In particular, when the initial PDF is strongly peaked at $x=x_{0}$, the marginal PDF of $x$, call it $p(x, t)$, has the following time evolution:

$$
p(x, t)=\sqrt{\frac{\beta}{\pi}} e^{-\beta(x-\langle x\rangle)^{2}},
$$

where $\langle x\rangle$ is the mean position, and $\beta$ is the inverse temperature given as

$$
\langle x\rangle=x_{0} e^{-\mu_{o} t}, \frac{1}{2 \beta}=\left\langle(x-\langle x\rangle)^{2}\right\rangle=\frac{D\left(1-e^{-2 \mu_{o} t}\right)}{2 \mu_{o}},
$$

In a long time limit, a PDF approaches a stationary Gaussian distribution where the variance of the PDF is set by $\left\langle x^{2}\right\rangle=$ $D / 2 \mu_{o}$. This stationary distribution can be linked to thermodynamic equilibrium distribution by the fluctuation-dissipation theorem by using $\frac{1}{2}\left\langle x^{2}\right\rangle=\frac{1}{2} T$ (with the Boltzmann constant $k_{B}=1$ ) and Eq. (4) as $t \rightarrow \infty$ as follows:

$$
T=\frac{1}{2 \beta(t \rightarrow \infty)}=\frac{D}{2 \mu_{o}},
$$

which is the Einstein's relation. We note that a factor of $1 / 2$ in the last term in Eq. (4) and (5) is due to our definition of $D$ in Eq. (2); that is, conventionally, Eq. (2) is defined with $2 D$ instead of $D$. For small time, the effect of the linear force is negligible compared to $\frac{d x}{d t}$, and the second equation in Eq. (4) recovers the usual Brownian motion with $\left\langle(x-\langle x\rangle)^{2}\right\rangle \propto t$. For large time, the linear force becomes important, leading to the approach to the stationary PDF. Mathematically, a Gaussian process offers a great analytical 
tractability in computing various statistical quantities (e.g., all even moments determined by second moments, etc).

Gaussian statistics, however, has serious limitations in explaining emerging complex phenomena such as anomalous transport (super- or sub-diffusion), intermittency, selforganisation, or phase transition where a long-range correlation plays a key role [4-21]. In fact, non-Gaussian PDFs often observed in these systems have stimulated active research on nonequilibrium statistics by considering nonlinear interaction, finite-correlated or Levy-flight noise, multiplicative noise, fractional calculus, etc. (e.g., see Refs. [4-15,17,19-23]). The main aim of this paper to shed light on this issue by elucidating the effect of nonlinear interaction during the relaxation process of an initial PDF to a final stationary PDF as an example of nonequilibrium processes.

To gain the key insight, it is valuable to consider the simplest nonlinear version of Eq. (1) that has the same symmetry property under $x \rightarrow-x$ as the $\mathrm{O}-\mathrm{U}$ process and compare results with the O-U process [e.g., Eq. (3)]. To this end, we consider a cubic nonlinear force $\left(\propto x^{3}\right)$ for a quartic potential $V(x)=\mu x^{4} / 4$ in Eq. (1) while keeping the same short-correlated noise $\xi$ given in Eq. (2). The cubic force has in fact been widely used to model enhanced diffusion in various systems, including mixing in stellar interiors (e.g., Ref. [24]) and self-organization of shear flow by generalizing a sand pile in a continuous limit [14,20,25]. In particular, Ref. [20] showed that similar stationary PDFs are obtained from a zerodimensional dynamical model, one- and two-dimensional (1D and 2D) fluid models. This suggests important implications of the results from a Langevin equation with cubic nonlinearity for a broad range of other physical problems with the same highest nonlinearity.

For the cubic process, the prediction of time-dependent PDFs has proven to be elusive [2]. However, a stationary distribution can easily be shown to be a quartic exponential PDF $p(x, t)=N e^{-\beta x^{4}}$ where $N \propto \beta^{-1 / 4}$ (see Appendix A for its property), to which any initial PDF relaxes in the long time limit. In this paper, we report on time-dependent PDFs using analytical and computational studies. Analytically, a path integral formulation [12,14-16,21,26-33] and a nonlinear time transformation are utilized to predict a transition probability. The PDF given in the path integral is computed by the saddle-point solution (instanton method) (e.g., see Refs. [14$16,21,27-29,31,32])$. The predicted $p(x, t)$ in general involves a time integral, and useful time-dependent PDFs with the explicit dependence on $x$ and $t$ are presented in certain limits (e.g., in the short and long time limits). Numerical simulations of the Fokker-Planck equation present detailed evolution of the time-dependent PDF and confirm our analytical predictions.

We note that instantons originated in quantum mechanics as a nonperturbative way of computing the transition amplitude from one ground state to another. The basic idea is that the uncertainty relationship between position and momentum allows one to formulate the transition amplitude from the initial to the final position by a path, and the transition amplitude from one ground state to another can be isolated by considering Euclidean action by taking time to be imaginary. An instanton is a saddle-point solution of Euclidean action and corresponds to one particular path that leads to the transition amplitude between ground states and was used in gauge field theory to compute the transition amplitude from one vacuum to another [31]. About 20 years later, the method was adapted to a classical fluid problem [32] and to a plasma problem [14-16]. In particular, Refs. [27-29] reported a series of detailed calculations for (multi)-instantons for double wells and different anharmonic potentials in quantum mechanics. Refs. [14-16] utilized it to predict stationary PDF tails of anomalous transport due to large events by taking a long-time limit, while Ref. [21] generalized this methodology to predict the time-dependent PDF for the O-U process without taking such a long-time limit. It is the purpose of this paper to extend this work further to nonlinear stochastic processes. The contribution of this work lies in the prediction of the time-dependent PDFs $p(x, t)$ by calculating the instanton solutions which satisfy the boundary conditions at the initial and final times (see Sec. II).

The remainder of the paper is as follows. We present the path integral solution of PDFs in Sec. II. Exact time-dependent PDF by simulations are provided in Sec. III. Section IV contains discussions and conclusions.

\section{ANALYTICAL PREDICTION}

We consider the evolution of a random variable $x$ under a quartic potential $V(x)=\mu x^{4} / 4$ in Eq. (1) and a white noise given by Eq. (2) as

$$
\frac{d x}{d t}=-\mu x^{3}+\xi
$$

where $\mu$ is the frictional constant for the cubic force. To gain a key insight into the evolution of this system, it is useful to obtain the equation for the mean and fluctuating components of $x$ by letting $x=\langle x\rangle+\delta x$ in Eq. (6). Here $\langle x\rangle$ and $\delta x$ are the mean value and fluctuation so that $\langle\delta x\rangle=0$. Then the time evolution of $\langle x\rangle$ and $\delta x$ can easily be shown to be as

$$
\begin{gathered}
\frac{d\langle x\rangle}{d t}=-\mu\left[\left(\langle x\rangle^{2}+\left\langle(\delta x)^{2}\right\rangle\right)\langle x\rangle+\left\langle(\delta x)^{3}\right\rangle\right], \\
\frac{d \delta x}{d t}=-3 \mu\langle x\rangle^{2} \delta x+G+\xi
\end{gathered}
$$

where $G=3\langle x\rangle\left[(\delta x)^{2}-\left\langle(\delta x)^{2}\right\rangle\right]+(\delta x)^{3}-\left\langle(\delta x)^{3}\right\rangle$. For small fluctuation, we can apply the quasilinear analysis to ignore $G$ and $\left\langle(\delta x)^{3}\right\rangle$ in Eqs. (7)-(8) and obtain approximate equations:

$$
\begin{gathered}
\frac{d\langle x\rangle}{d t}=-\mu\left[\langle x\rangle^{2}+\left\langle(\delta x)^{2}\right\rangle\right]\langle x\rangle, \\
\frac{d \delta x}{d t}=-3 \mu\langle x\rangle^{2} \delta x+\xi \equiv-\mu_{o} \delta x+\xi,
\end{gathered}
$$

where $\mu_{o}=3 \mu\langle x\rangle^{2}$ is the (linear) force constant for $\delta x$. When fluctuations are negligible compared to the mean value $\left[\left\langle(\delta x)^{2}\right\rangle \ll\langle x\rangle^{2}\right]$, Eq. (9) becomes $\frac{d\langle x\rangle}{d t}=-\mu\langle x\rangle^{3}$, with the solution $\langle x\rangle=x_{0} / \sqrt{1+2 \mu x_{0}^{2} t}$ given the initial condition $\langle x(t=0)\rangle=x_{0}$. As the fluctuation $\delta x$ increases in time, the second term in Eq. (9) gives the enhanced force, leading to the faster movement of the PDF peak from $x_{0}$ towards $x=0$ (see Sec. III for more details). On the other hand, Eq. (10) shows that the evolution of fluctuation $\delta x$ is a linear O-U process with the effective linear force $\mu_{o}=3 \mu\langle x\rangle^{2}$. 
For analytical computation of a time-dependent PDF, we consider a narrow initial PDF approximated by the $\delta$ function $p(x, t=0)=\delta\left(x-x_{0}\right)$. In order to obtain a PDF at any later time, we utilize a path integral formulation by expressing the transition probability $p\left(x_{f}, t_{f} ; x_{0}, 0\right)$ between initial $x_{0}$ at $t=0$ and $x_{f}$ at later time $t=t_{f}[12-16,21,26,30,33]$ as follows:

$$
p\left(x_{f}, t_{f} ; x_{0}, 0\right) \propto \int_{\left(x_{0}, 0\right)}^{\left(x_{f}, t_{f}\right)} D x[t] D \bar{x}[t] e^{-S},
$$

where $S$ is the action given by (see Appendix B)

$$
S=\int_{t_{0}}^{t_{f}} d t\left[-i\left(\frac{d x}{d t}+\mu x^{3}\right) \bar{x}+\frac{1}{2} D \bar{x}^{2}+\psi(x)\right] .
$$

Here $\bar{x}$ is the conjugate variable to $x$ [e.g., see Eq. (B3) and also Eqs. (3)-(5) in Ref. [16]], which effectively captures the effect of the stochastic forcing $\xi \cdot \psi=\frac{3}{2} \mu x^{2}$ in Eq. (12) is due to the coordinate transformation between $\xi$ and $x$. As $\psi$ is negligible in the limit of small $D$ [12], we drop this term for analytical tractability below. The transition probability given in Eq. (11) involves the integration along all the paths connecting initial $\left(x_{0}, 0\right)$ and final $\left(x_{f}, t_{f}\right)$ points. We evaluate Eq. (11) approximately to leading order in $D$ by finding a particular path which makes the largest contribution to the action $S$. This is the so-called saddle-point solution (instantons) which minimizes the action $S$, satisfying the zero variation of $S$ with respect to $x$ and $\bar{x}$ as follows:

$$
\begin{aligned}
& \frac{\delta S}{\delta \bar{x}}=0 \rightarrow i\left(\frac{d x}{d t}+\mu x^{3}\right)=D \bar{x} \\
& \frac{\delta S}{\delta x}=0 \rightarrow i\left(\frac{d \bar{x}}{d t}-3 \mu \bar{x}^{2} x\right)=0 .
\end{aligned}
$$

Equations (13)-(14) are to be solved with the boundary conditions $x(t=0)=x_{0}$ and $x\left(t=t_{f}\right)=x_{f}$. We note that the crux of the instanton method is to capture a nontrivial "timevarying state" as our basic state. As noted in the introduction, instanton solutions satisfying boundary conditions were used for the computation of the time-dependent PDFs for the O-U process [21].

The explicit appearance of the conjugate variable $\bar{x}$ in Eqs. (13)-(14) reflects a vital role of the stochastic forcing in the determination of a saddle-point solution. That is, the leading order contribution to the action cannot be obtained by simply ignoring $\xi$ in Eq. (1). Saddle-point solutions $x_{s}$ and $\bar{x}_{s}$ to Eqs. (13)-(14) then give the effective action $S_{\text {eff }}$, simplifying Eq. (11) as follows:

$$
p\left(x_{f}, t_{f} ; x_{0}, 0\right)=N \exp \left[-S_{\mathrm{eff}}\right],
$$

where $N$ is a normalization constant to satisfy $\int d x_{f} p\left(x_{f}, t_{f} ; x_{0}, 0\right)=1$ and $S_{\text {eff }}$ is the effective action

$$
\begin{aligned}
S_{\mathrm{eff}} & =\int_{0}^{t_{f}} d t \frac{1}{2 D}\left(\frac{d x_{s}}{d t}+\mu x_{s}^{3}\right)^{2} \\
& =-\int_{0}^{t_{f}} d t \frac{D}{2} \bar{x}_{s}^{2} .
\end{aligned}
$$

In the following, in order to obtain saddle-point solutions to Eqs. (13)-(14), we introduce a nonlinear (nonlocal) time $\tau$ as

$$
\tau(t)=\int_{0}^{t} d t_{1}\left[x\left(t_{1}\right)\right]^{2}
$$

and recast Eqs. (13)-(14) in terms of $\tau$ as follows:

$$
\begin{gathered}
\frac{1}{3} \frac{d}{d \tau}\left[x^{3} e^{3 \mu \tau}\right]=-i D \bar{x} e^{3 \mu \tau}, \\
\frac{d}{d \tau}\left[\bar{x} e^{-3 \mu \tau}\right]=0,
\end{gathered}
$$

by using $\frac{d}{d t}=x^{2} \frac{d}{d \tau}$. See Appendix C for an example of how the transformation (18) works. The solutions to Eqs. (19)-(20) are found as

$$
\begin{gathered}
\bar{x}(t)=\bar{x}(t=0) e^{3 \mu \tau}, \\
(x(t))^{3} e^{3 \mu \tau}=x_{0}^{3}+B\left[e^{6 \mu \tau}-1\right],
\end{gathered}
$$

where

$$
B=-i \frac{D \bar{x}(t=0)}{2 \mu} .
$$

The boundary condition $x\left(t=t_{f}\right)=x_{f}$ fixes the value of $B$ as

$$
B=\frac{x_{f}^{3} e^{3 \mu \tau_{f}}-x_{0}^{3}}{e^{6 \mu \tau_{f}}-1},
$$

where $\tau_{f}=\tau\left(t=t_{f}\right)$. In terms of $x(\tau), t$ is determined by the inverse of Eq. (18) as

$$
t=\int_{0}^{\tau} d \tau_{1} \frac{1}{\left[x\left(\tau_{1}\right)\right]^{2}},
$$

while the effective action $S_{\text {eff }}$ in Eq. (17) is expressed by

$$
S_{\text {eff }}=\frac{2 \mu^{2} B^{2}}{D} \int_{0}^{\tau_{f}} d \tau_{1} \frac{e^{6 \mu \tau_{1}}}{\left[x\left(\tau_{1}\right)\right]^{2}},
$$

where Eqs. (21), (23), and (24) are used. In the following two subsections, we separately consider the cases $x_{0}=0$ and $x_{0} \neq 0$.

$$
\text { A. } x_{0}=0
$$

For $x_{0}=0$, the time evolution of the PDF involves only a change in its width until it becomes the stationary exponential PDF in the long time limit. Equation (22) is simplified in this case as

$$
\begin{aligned}
{[x(\tau)]^{3} } & =B_{0}\left(e^{3 \mu \tau}-e^{-3 \mu \tau}\right), \\
B_{0} & =\frac{x_{f}^{3} e^{3 \mu \tau_{f}}}{e^{6 \mu \tau_{f}}-1} .
\end{aligned}
$$

With the help of Eq. (27), Eq. (25) can be expressed as

$$
t_{f}=\frac{1}{2 \mu B_{0}^{\frac{2}{3}}} \int_{0}^{z_{f}} d z_{1} \frac{1}{\left(1-z_{1}^{3}\right)^{\frac{2}{3}}}=\frac{1}{2 \mu B_{0}^{\frac{2}{3}}} \sin _{\frac{3}{2}, 3}^{-1}\left(z_{f}\right) .
$$

Here $z_{1}=\left(1-e^{-6 \mu \tau_{1}}\right)^{\frac{1}{3}}$ and $z_{f}=\left(1-e^{-6 \mu \tau_{f}}\right)^{\frac{1}{3}} \cdot \sin _{\frac{3}{2}, 3}^{-1}\left(z_{f}\right)$ is the generalized $p, q$-family inverse sine function (e.g., see 
Ref. [34] and Appendix D) with $p=\frac{3}{2}$ and $q=3$, which satisfy the generalized trigonometric identity

$$
\left|\sin _{p, q}\left(z_{f}\right)\right|^{q}+\left|\cos _{p, q}\left(z_{f}\right)\right|^{p}=1 .
$$

Equation (28) thus gives us the expression for $z_{f}$ in terms of time $t_{f}$ as

$$
z_{f}=\sin _{\frac{3}{2}, 3}\left(2 \mu B_{0}^{\frac{2}{3}} t_{f}\right) .
$$

On the other hand, the effective action $S_{\text {eff }}$ in Eq. (26) can be put into the following form:

$$
\begin{aligned}
S_{\text {eff }} & =\frac{\mu B_{0}^{\frac{4}{3}}}{D} \int_{0}^{z_{f}} d z_{1} \frac{1}{\left(1-z_{1}^{3}\right)^{\frac{5}{3}}} \\
& =\frac{\mu B_{0}^{\frac{4}{3}}}{2 D}\left[2 \mu B_{0}^{\frac{2}{3}} t_{f}+\frac{z_{f}}{\left(1-z_{f}^{3}\right)^{\frac{2}{3}}}\right],
\end{aligned}
$$

where we used Eq. (28) and the following identity (see Appendix E):

$$
\int_{0}^{z} d z_{1}\left(1-z_{1}^{3}\right)^{-5 / 3}=\frac{1}{2}\left[\int_{0}^{z} d z_{1}\left(1-z_{1}^{3}\right)^{-2 / 3}+z\left(1-z^{3}\right)^{-2 / 3}\right] .
$$

By using Eq. (27), we rewrite Eq. (31) as

$$
S_{\mathrm{eff}}=\frac{\mu}{2 D}\left[2 \mu B_{0}^{2} t_{f}+\frac{x_{f}^{4}}{\left(1-e^{-6 \mu \tau_{f}}\right)}\right] .
$$

The substitution of Eq. (33) into Eq. (15) then determines how PDFs vary with $x_{f}$ depending on $t_{f}$. The normalization factor $N=N(t)$ in Eq. (15) alters the overall amplitude of the PDF and is discussed in Sec. II C.

Equation (33) involves $\tau_{f}$ and $B_{0}$, which is a function of $\tau_{f}$. Since $\tau_{f}=\int_{0}^{t_{f}} d t_{1}\left[x\left(t_{1}\right)\right]^{2}, p(x, t)$ is not given by a simple function of $x$ and $t$ but as an integral.

In the short and long time limits, the PDFs can, however, be approximated by a function depending on $x$ and $t$. To demonstrate this, we examine the behavior of $t$ and $S_{\text {eff }}$ in Eqs. (28) and (33) in the short and long time limits, respectively. First, in the long time limit $\left(z_{f} \rightarrow 1\right), \sin _{\frac{3}{2}, 3}^{-1}\left(z_{f}\right) \rightarrow \frac{1}{2} \pi_{\frac{3}{2}, 3}$, where $\pi_{\frac{3}{2}, 3}$ is the generalized $\pi_{p, q}$ (see Appendix D). Thus, Eqs. (28) and (33) give us to leading order in $1 / t_{f}$

$$
\begin{aligned}
z_{f}^{3} & \sim 1-\left(\frac{\pi_{\frac{3}{2}, 3}}{4 \mu t_{f} x_{f}^{2}}\right)^{3}, \\
S_{\mathrm{eff}} & \sim \frac{\mu}{2 D} x_{f}^{4}\left[1+\left(\frac{\pi_{\frac{3}{2}, 3}}{4 \mu t_{f} x_{f}^{2}}\right)^{3}\right] .
\end{aligned}
$$

Equation (34) shows how the PDF evolves into the quartic exponential PDF in a long time limit. We can estimate the time required to reach the stationary state by examining the behavior of the PDF width in Eq. (34). To do this, we let $\alpha=\pi_{\frac{3}{2}, 3} /\left(4 \mu t_{f}\right)$ and find $x_{f}$ for $S_{e f f}=1$ (i.e., the width at half-peak):

$$
\frac{\mu}{2 D}\left[x_{f}^{4}+\frac{\alpha^{3}}{x_{f}^{2}}\right]=1
$$

Solving the above by perturbation as $x_{f}=x_{f}^{(0)}+x_{f}^{(1)}+\cdots$ for small $\alpha \ll 1$ leads us to

$$
x_{f} \sim\left(\frac{\mu}{2 D}\right)^{\frac{1}{4}}\left[1-\left(\frac{\mu}{4 D}\right)^{\frac{3}{2}}\left(\frac{\pi_{\frac{3}{2}, 3}}{4 \mu t_{f}}\right)^{3}\right] \text {. }
$$

The leading order solution $x_{f}^{(0)}=(\mu / 2 D)^{1 / 4}$ represents the width of the stationary PDF, which is much wider than the width $\propto D^{-1 / 2}$ in the case of the linear O-U process for small $D$. From the condition that the second, time-dependent, term is much smaller than the first term in the above, we obtain an estimate for the critical time $t_{c}$ required to reach the stationary state:

$$
t_{f}>\frac{\pi_{\frac{3}{2}, 3}}{8 \sqrt{\mu D}}\left(\equiv t_{c}\right)
$$

Interestingly, the critical time $t_{c}$ increases as $D^{-1 / 2}$ as $D$ decreases. That is, the smaller the diffusion, the longer the relaxation time $t_{c}$. This should be contrasted to the linear friction case where the relaxation time is independent of $D$ and solely determined by the friction $\mu$. This dependence of the relaxation time on $D$ reveals one of the important characteristics of the nonlinear process where the diffusion sets not only the spatial structure (width), but also the time structure.

On the other hand, in a short time limit $\left(z_{f} \rightarrow 0\right)$, we evaluate $\sin _{\frac{3}{2}, 3}^{-1}\left(z_{f}\right) \sim z_{f}+\frac{1}{6} z_{f}^{4}$ and use $B_{0} z_{f}^{3}=x_{f}^{3}\left(1-z_{f}^{3}\right)^{1 / 2}$ to obtain

$$
\begin{aligned}
z_{f}^{6}\left[\sin _{\frac{3}{2}, 3}^{-1}\left(z_{f}\right)\right]^{3} & =z_{f}^{6}\left(z_{f}+\frac{1}{6} z_{f}^{4}\right)^{3} \\
& =\left(2 \mu t_{f} x_{f}^{2}\right)^{3}\left(1-z_{f}^{3}\right) .
\end{aligned}
$$

We can then find the leading order solution to Eq. (36) for small $2 \mu t x_{f}^{2}$ in the following form

$$
\begin{aligned}
& z_{f}^{3} \sim\left(2 \mu t x_{f}^{2}\right)\left(1-\mu t x_{f}^{2}\right), \\
& B_{0}^{2}=\frac{x_{f}^{6}\left(1-z_{f}^{3}\right)}{z_{f}^{6}} \sim \frac{x_{f}^{6}\left(1+2 \mu t_{f} x_{f}^{2}\right)}{\left(2 \mu t_{f} x_{f}^{2}\right)^{2}},
\end{aligned}
$$

thereby obtaining

$$
S_{\mathrm{eff}} \sim \frac{x_{f}^{2}}{2 D t_{f}}\left[1+\frac{3}{2} \mu t_{f} x_{f}^{2}\right]
$$

The parameter $2 \mu t x_{f}^{2}$ physically represents the effect of a nonlinear damping, and the limit of the small value of this parameter corresponds to considering a sufficiently small time during which the effect of nonlinear damping can be considered to be small and thus computed as a small perturbation from no damping case.

The leading order behavior $S_{\text {eff }} \sim x_{f}^{2} / 2 D t_{f}$ reveals that initially, the evolution of the PDF is governed by the Gaussian process with the Gaussian PDF. The quartic term $x_{f}^{4}$ in Eq. (37) is symptomatic of the tendency towards a quartic (stationary) 
PDF in the long time limit, shown in Eq. (34). For $x_{0}=0$ considered in this subsection, the relaxation of a transient PDF undergoes two stages: the first is the Gaussian evolution where the white noise causes the Brownian motion with a negligible effect of the nonlinear force. When the nonlinear force becomes sufficiently large, the PDF broadens its width, settling in to the stationary quartic exponential.

\section{B. $x_{0} \neq 0$}

Unlike the case $x_{0}=0$, the time evolution of the PDF is governed by the movement of the peak of the PDF from $x_{0}$ to $x=0$ as well as the broadening of the PDF. Consequently, the relaxation of a transient PDF is more complex compared with the $x_{0}=0$ case considered in the previous subsection. In the following, we show that the relaxation of a transient PDF involves one more stage between the initial Gaussian evolution and the final settling in to the quartic exponential PDF. The extra stage appears when the nonlinear force becomes sufficiently large and leads to a linear force with the effective force coefficient $3 \mu\langle x\rangle^{2}$ for fluctuations. That is, the second Gaussian evolution is approximately the O-U process with the effective linear force $\mu_{0}=3 \mu\langle x\rangle^{2}$, similar to the quasilinear result in Eq. (10).

In order to obtain the PDF, we need to find $S_{\text {eff }}$ in Eq. (15) via Eqs. (26), (25), and (22). To this end, it is convenient to rewrite Eq. (22) in the following form:

$$
x^{3}=B e^{3 \mu \tau}+\alpha e^{-3 \mu \tau}=B e^{-3 \mu \tau}\left[e^{6 \mu \tau}-\gamma\right],
$$

where $B$ is given by Eq. (24) and

$$
\begin{aligned}
& \alpha=x_{0}^{3}-B=\frac{x_{0}^{3} e^{6 \mu \tau_{f}}-x_{f}^{3} e^{3 \mu \tau_{f}}}{e^{6 \mu \tau_{f}}-1}, \\
& \gamma=-\frac{\alpha}{B}=\frac{x_{0}^{3} e^{6 \mu \tau_{f}}-x_{f}^{3} e^{3 \mu \tau_{f}}}{x_{0}^{3}-x_{f}^{3} e^{3 \mu \tau_{f}}} .
\end{aligned}
$$

With no loss of generality, we take $x_{0} \geqslant 0$ in this paper since the symmetry of our cubic system under $x \rightarrow-x$ guarantees exactly equivalent results for $x_{0}<0$. Equation (39) reveals the following three different cases depending on the sign of $\gamma$ and the ratio of $x_{f}$ to $x_{0}$ :

$$
\begin{aligned}
& \text { CASE1: } \gamma>e^{6 \mu \tau_{f}}\left(x_{f}<e^{-\mu \tau_{f}} x_{0}\right) \\
& \text { CASE2: } \gamma<0\left(e^{-\mu \tau_{f}} x_{0}<x_{f}<e^{\mu \tau_{f}} x_{0}\right) \\
& \text { CASE3: } 0<\gamma<e^{6 \mu \tau_{f}}\left(x_{f}>e^{\mu \tau_{f}} x_{0}\right) .
\end{aligned}
$$

When $x_{0}=0, \gamma=1$, recovering the case in the previous subsection and thus CASE2 becomes irrelevant. That is, $x_{0} \neq$ 0 leads to the appearance of CASE2. For $x_{0}>0$, a schematic diagram for the different cases is shown in Fig. 1 where $x_{f}$ is plotted against $\tau_{f}$. From Fig. 1, we observe that a short time behavior is described by CASE1/CASE3 while a long time behavior is obtained from CASE2. As $\tau_{f}$ increases from zero, the region for CASE1/CASE3 gradually decreases while the region for CASE2 increases. The crossover between CASE1 and CASE2 is set by $x_{f}=x_{0} e^{-\mu \tau_{f}} \equiv x_{c}$ while the crossover between CASE2 and CASE3 is set by $x_{f}=x_{0} e^{\mu \tau_{f}} \equiv x_{b}$. Here $x_{c}=x_{0} e^{-\mu \tau_{f}}$ is the characteristic of the movement of the PDF peak. Consequently, the left side of the PDF peak is described

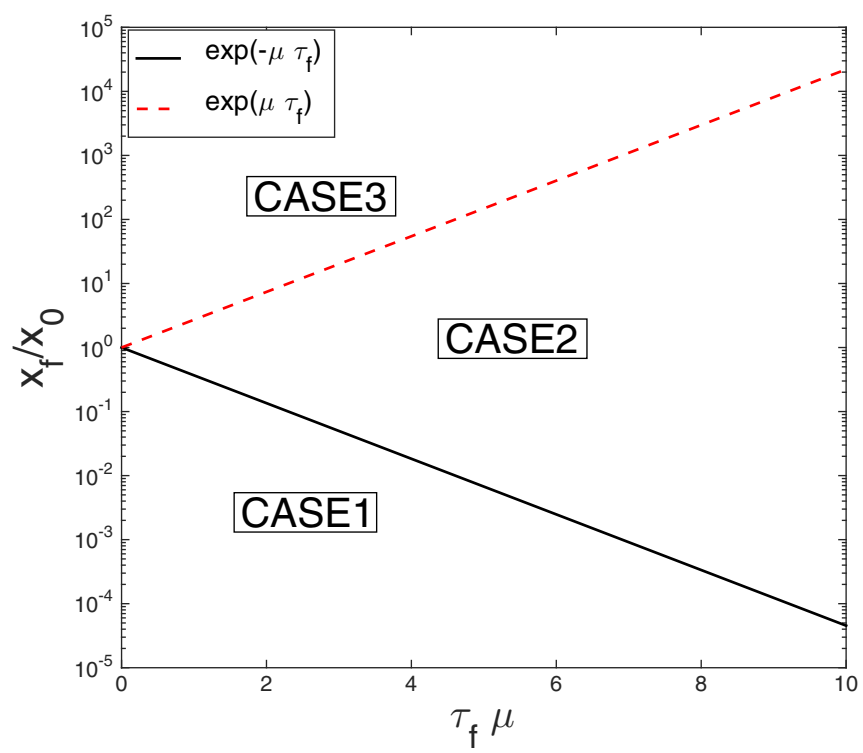

FIG. 1. A schematic diagram of $x_{f} / x_{0}$ against $\mu \tau_{f}$.

by CASE1 while the right side of the PDF peak by CASE2. On the other hand, $x_{b}=x_{0} e^{\mu \tau_{f}}$ characterizes the boundary around which the PDF becomes negligible as $S_{\text {eff }}$ becomes very large (details not shown here). That is, $x_{b}$ sets the boundary between the region of a small probability due to a stochastic noise and a dead zone. (That is, the probability is zero for $x_{f}>x_{b}$ in CASE3.) To appreciate the meaning of $\gamma$, we rewrite

$$
\gamma=\frac{x_{b}^{3}-x_{f}^{3}}{x_{c}^{3}-x_{f}^{3}} \equiv \frac{\Delta_{b}}{\Delta},
$$

where $\Delta=x_{c}-x_{f}$ and $\Delta_{b}=x_{b}-x_{f}$. Since $\Delta$ and $\Delta_{b}$ measure the deviation of $x_{f}$ from $x_{c}$ and $x_{b}$, respectively, the probability becomes larger for smaller $\Delta$ and larger $\Delta_{b}$. For instance, CASE1 with the large value of $\gamma$ corresponds to the region where the probability is largest.

To determine the relation between $t_{f}$ and $\tau_{f}$ along $x_{f}=$ $x_{0} e^{-\mu \tau_{f}}$, we note that Eq. (39) gives $\alpha=x_{0}^{3}$ and $B=0$. Thus, the saddle-point solution in Eq. (38) is simplified as $x=x_{0} e^{-\mu \tau}$, leading to $t_{f}$ in Eq. (25):

$$
\begin{aligned}
t_{f} & =\int_{0}^{\tau_{f}} d \tau_{1} \frac{1}{\left[x\left(\tau_{1}\right)\right]^{2}}=\frac{1}{2 \mu x_{0}^{2}}\left(e^{2 \mu \tau_{f}}-1\right) \\
& =\frac{1}{2 \mu x_{0}^{2}}\left(\frac{x_{0}^{2}}{x_{f}^{2}}-1\right),
\end{aligned}
$$

and consequently

$$
e^{2 \mu \tau_{f}}=\frac{x_{0}^{2}}{x_{f}^{2}}=1+2 \mu t_{f} x_{0}^{2} .
$$

In the following subsections, $\gamma>0$ (CASE1) and $\gamma<0$ (CASE2) are separately considered. (The analysis for $y-\gamma>$ 0 is included in Appendix $\mathrm{F}$ for completeness.)

$$
\text { 1. CASE1: } \gamma \geqslant 0(\alpha>0, B<0), x_{f}<x_{0} e^{-\mu \tau_{f}}
$$

We first find the relation between $t_{f}$ and $\tau_{f}$ by using Eq. (38) in Eq. (25) for $\gamma>0$. To do this, we let $y=e^{6 \mu \tau}$ and note 
$\gamma-y>0$ (since $\left.\gamma-e^{6 \mu \tau_{f}}>0\right)$ to obtain

$$
t_{f}=\frac{1}{6 \mu B^{\frac{2}{3}}} I_{0},
$$

where

$$
I_{0}=\int_{1}^{y_{f}} \frac{d y}{y^{\frac{2}{3}}} \frac{1}{(\gamma-y)^{\frac{2}{3}}},
$$

and $y_{f}=e^{6 \mu \tau_{f}}$. The integral in Eq. (42) can be written in terms of the generalized two-family inverse hyperbolic sine (e.g., see Ref. [34]). On the other hand, Eq. (26) can be shown as

$$
\begin{aligned}
S_{\mathrm{eff}} & =\frac{\mu B^{\frac{4}{3}}}{3 D} I_{1} \\
& =\frac{\mu}{6 D}\left\{6 \mu t_{f}|\alpha B|-3|B|^{\frac{4}{3}}\left[y^{\frac{1}{3}}(\gamma-y)^{\frac{1}{3}}\right]_{1}^{y_{f}}\right\},
\end{aligned}
$$

where

$$
I_{1}=\int_{1}^{y_{f}} d y \frac{y^{\frac{1}{3}}}{(\gamma-y)^{\frac{2}{3}}} .
$$

In the second line in Eq. (43), we used Eq. (41) and the identity (see Appendix G):

$$
I_{1}=\frac{1}{2}\left\{\gamma I_{0}-3\left[y^{\frac{1}{3}}(\gamma-y)^{\frac{1}{3}}\right]_{1}^{y_{f}}\right\} .
$$

Equation (43) shows that $S_{\text {eff }}$ becomes zero along the characteristics $x_{f}=x_{0} e^{-\mu \tau_{f}}$ as $B=0$. Recall that along these characteristics, Eq. (40) holds. This means that the PDF takes its maximum value along these characteristics. To facilitate the analysis, it is thus useful to consider the deviation $\Delta$ from the characteristics

$$
\Delta=x_{0} e^{-\mu \tau_{f}}-x_{f},
$$

where $\Delta \geqslant 0$ since $x_{f} \leqslant x_{0} e^{-\mu t_{f}}$. By using Eqs. (24), (39), and (44) in Eq. (43), we obtain

$$
\begin{aligned}
S_{\mathrm{eff}} & =\frac{\mu}{2 D}|B|\left[2 \mu t_{f}\left(x_{0}^{3}+|B|\right)-\left(x_{f} e^{3 \mu \tau_{f}}-x_{0}\right)\right] \\
& =\frac{\mu}{2 D}|B|\left[2 \mu t_{f}|B|+\Delta e^{3 \mu \tau_{f}}\right],
\end{aligned}
$$

where $\quad \alpha=x_{0}^{3}+|B| \quad($ as $\quad B<0), \quad\left[y^{\frac{1}{3}}(\gamma-y)^{\frac{1}{3}}\right]_{1}^{y_{f}}=$ $\left(x_{f} e^{3 \mu \tau_{f}}-x_{0}\right)|B|^{-\frac{1}{3}}$, and $y_{f}=e^{6 \mu \tau_{f}}$ are used. To obtain the PDF in terms of $\Delta$, we rewrite $|B|$ by using Eq. (46) and $Q=y_{f}^{\frac{1}{3}}=e^{2 \mu \tau_{f}}=1+2 \mu t_{f} x_{0}^{2}$ as

$$
|B|=\frac{1}{2 \mu t_{f}} \frac{3 \Delta Q^{\frac{1}{2}}-3 \frac{\Delta^{2}}{x_{0}} Q+\frac{\Delta^{3}}{x_{0}^{2}} Q^{\frac{3}{2}}}{Q^{2}+Q+1} .
$$

Then, by using Eq. (48) together with $Q=1+2 \mu t_{f} x_{0}^{2}$ from Eq. (40) in Eq. (46), we obtain the leading order behavior of $S_{\text {eff }}$ in the short and long time limits depending on the value of $Q$, respectively, as follows:

$$
\begin{aligned}
S_{\mathrm{eff}}= & \frac{\Delta^{2}}{4 D t_{f}}\left[2-3 \frac{\Delta}{x_{0}}+2 \frac{\Delta^{2}}{x_{0}^{2}}\right], \quad(\text { for } Q \rightarrow 1) \\
\sim & \frac{\mu \Delta^{2}}{2 D}\left[\Delta^{2}\left(1-1.5 Q^{-1}\right)\right. \\
& \left.-3 x_{0} \Delta Q^{-\frac{1}{2}}+3 x_{0}^{2} Q^{-1}\right] . \quad(\text { for } \quad Q \gg 1)
\end{aligned}
$$

The crossover between Eqs. (49) and (50) is set by the time where $e^{\mu \tau_{f}} \sim 1$, or equivalently, $t_{f} \ll\left(2 \mu x_{0}^{2}\right)^{-1}$, independently of $D$. In the short time limit $(Q \rightarrow 1)$, the first term in Eq. (49) demonstrates the Gaussian PDF, the width increasing as $t^{1 / 2}$ for small $\Delta$. As the width broadens, the cubic and quartic terms in $\Delta$ become important for the PDF. In particular, the quartic term leads to the broadening of the PDF width just before the transition to the second stage governed by Eq. (50) for a sufficiently large time $Q \gg 1$.

Notably, Eq. (50) reveals the mixture of the Gaussian PDF, cubic, and and quartic exponential PDF depending on the relative size of the three terms on the right-hand side. Since $x_{0}^{2} Q^{-1} \sim\left\langle x^{2}\right\rangle$, we recognize that $3 \mu x_{0}^{2} Q^{-1}=3 \mu\left\langle x^{2}\right\rangle=\mu_{o}$ is the effective linear force coefficient for fluctuations defined in Eq. (10). Therefore, the third term on the right of Eq. (50) gives rise to a Gaussian PDF with the inverse temperature $\propto \mu_{o} / 2 D$ (equivalently, with the width $\propto \sqrt{2 D / \mu_{o}}$ ). This is remarkably similar to the quasilinear result and $t^{1 / 2}$ increase in the PDF is caused by the decrease in $\mu_{o}$ as the mean value $\langle x\rangle$ decreases with the movement of the PDF peak towards $x=0$.

In comparison, the first term on the right of Eq. (50) leads to a quartic exponential PDF with the width $\propto(2 D / \mu)^{1 / 4}$ for larger time $Q^{-1} \rightarrow 0$. To compare the size of the first and third term on the right of Eq. (50), we approximate $Q^{-1} \sim 2 \mu x_{0} t_{f}$ and $\Delta \sim 2 D t_{f}$ to obtain the estimate of the ratio of the third to the first terms as

$$
\frac{3 x_{0}^{2} Q^{-1}}{\Delta^{2}\left(1-Q^{-1}\right)} \sim \frac{3}{4 t_{f}^{2} D \mu} .
$$

Thus, for $t_{f} \ll \sqrt{3 / 4 D \mu}$, the PDF is Gaussian, while in the opposite large time, the PDF relaxes to the quartic exponential PDF. This transition time from the Gaussian to stationary quartic exponential PDF depends on $D$, increasing as $D$ becomes smaller.

Finally, it is interesting to observe that in both Eqs. (49) and (50), the first order correction term has a negative sign (since $\Delta>0$ ), leading to the increase in the PDF above the leading order term. This is to be contrasted to CASE2 considered in the next subsection where the first order correction $\Delta$ is shown to depress the PDF (as $S_{\text {eff }}$ increases).

\section{CASE2: $\gamma<0(\alpha>0, B>0), x_{0} e^{-\mu \tau_{f}}<x_{f}<x_{0} e^{\mu \tau_{f}}$}

We begin by rewriting Eq. (38) as

$$
x=B^{\frac{1}{3}} e^{-\mu \tau}\left[e^{6 \mu \tau}+|\gamma|\right]^{\frac{1}{3}},
$$

where $B(>0)$ is given by Eq. (24). $I_{0}$ in Eq. (41) is given by

$$
I_{0}=\int_{1}^{y_{f}} \frac{d y}{y^{\frac{2}{3}}} \frac{1}{(y+|\gamma|)^{\frac{2}{3}}},
$$

where $y=e^{6 \mu t}$ and $y_{f}=e^{6 \mu \tau_{f}}$. Similarly, Eq. (26) can be written as

$$
\begin{aligned}
S_{\mathrm{eff}} & =\frac{\mu B^{\frac{4}{3}}}{3 D} I_{1} \\
& =\frac{\mu}{2 D}\left\{-2 \mu t_{f}|\alpha B|+B^{\frac{4}{3}}\left[y^{\frac{1}{3}}(y+|\gamma|)^{\frac{1}{3}}\right]_{1}^{y_{f}}\right\},
\end{aligned}
$$


where

$$
\begin{aligned}
I_{1} & =\int_{1}^{y_{f}} d y \frac{y^{\frac{1}{3}}}{(y+|\gamma|)^{\frac{2}{3}}} \\
& =\frac{1}{2}\left\{-|\gamma| I_{0}+3\left[y^{\frac{1}{3}}(y+|\gamma|)^{\frac{1}{3}}\right]_{1}^{y_{f}}\right\} .
\end{aligned}
$$

As $x_{f}>x_{0} e^{-\mu \tau_{f}}$ in CASE2, we define $\Delta_{c}$ to be the deviation from the characteristics according to

$$
x_{f}=x_{0} e^{-\mu \tau_{f}}+\Delta_{c},
$$

where $\Delta_{c} \geqslant 0$. By using Eqs. (24), (39), and (46) in Eq. (53), we obtain

$$
\begin{aligned}
S_{\mathrm{eff}} & =\frac{\mu}{2 D} B\left[-2 \mu t_{f}\left(x_{0}^{3}-B\right)+\left(x_{f} e^{3 \mu \tau_{f}}-x_{0}\right)\right] \\
& =\frac{\mu}{2 D}|B|\left[2 \mu t_{f} B+\Delta_{c} e^{3 \mu \tau_{f}}\right],
\end{aligned}
$$

where $\alpha=x_{0}^{3}-B(B>0),\left[y^{1 / 3}(\gamma-y)^{\frac{1}{3}}\right]_{1}^{y_{f}}=\left(x_{f} e^{3 \mu \tau_{f}}-\right.$ $\left.x_{0}\right) B^{-1 / 3}$, and $y_{f}=e^{6 \mu \tau_{f}}$ are used. To obtain the PDF in terms of $\Delta_{c}$, we again express $B$ by using Eq. (56) and $Q=y_{f}^{\frac{1}{3}}=$ $e^{2 \mu \tau_{f}}=1+2 \mu t_{f} x_{0}^{2}$ as

$$
B=\frac{1}{2 \mu t_{f}} \frac{3 \Delta_{c} Q^{\frac{1}{2}}+3 \frac{\Delta_{c}^{2}}{x_{0}} Q+\frac{\Delta_{c}^{3}}{x_{0}^{2}} Q^{\frac{3}{2}}}{Q^{2}+Q+1} .
$$

Then, by using Eq. (57) and $Q=1+2 \mu t_{f} x_{0}^{2}$ in Eq. (56), we obtain the leading order behavior of $S_{\text {eff }}$ in the short and long time limit as follows:

$$
\begin{aligned}
S_{\mathrm{eff}}= & \frac{\Delta_{c}^{2}}{4 D t_{f}}\left[2+3 \frac{\Delta_{c}}{x_{0}}+2 \frac{\Delta_{c}^{2}}{x_{0}^{2}}\right] \quad(\text { for } Q \rightarrow 1), \\
= & \frac{\mu \Delta_{c}^{2}}{2 D}\left[\Delta_{c}^{2}\left(1-1.5 Q^{-1}\right)\right. \\
& \left.+3 x_{0} \Delta_{c} Q^{-\frac{1}{2}}+3 x_{0}^{2} Q^{-1}\right] \quad(\text { for } Q \gg 1) .
\end{aligned}
$$

Similarly to Eqs. (49) and (50) in CASE1, the crossover between Eqs. (58) and (59) is set by the time where $e^{\mu \tau_{f}} \sim 1$, or equivalently, $t_{f} \sim\left(2 \mu x_{0}^{2}\right)^{-1}$, independently of $D$. In the short time limit ( $Q \rightarrow 1$ ), Eq. (58) demonstrates the initial Gaussian PDF, which becomes modified by the quartic term just before the transition to the second stage governed by Eq. (59). The second stage in Eq. (59) starts with another Gaussian evolution, followed by the transition from this Gaussian to the final stationary quartic exponential PDF for $t_{f} \ll \sqrt{3 / 4 D \mu}$ in the long time limit. In contrast to CASE1, in both limits, the first order correction which is odd in $\Delta_{c}>0$ is now positive and results in further decrease in the PDF as $\Delta_{c}$ increases.

\section{Summary of CASE1 and CASE2}

By combining Eqs. (49)-(50) and (58)-(59), we write $S_{\text {eff }}$ on either side of the PDF peak in terms of $\Delta_{c}=x_{f}-e^{-\mu \tau_{f}} x_{0}$ as

$$
\begin{aligned}
S_{\mathrm{eff}}= & \frac{\Delta_{c}^{2}}{4 D t_{f}}\left[2+3 \frac{\Delta_{c}}{x_{0}}+2 \frac{\Delta_{c}^{2}}{x_{0}^{2}}\right] \quad(\text { for } Q \rightarrow 1), \\
= & \frac{\mu \Delta_{c}^{2}}{2 D}\left[\Delta_{c}^{2}\left(1-1.5 Q^{-1}\right)\right. \\
& \left.+3 \Delta_{c} x_{0} Q^{-\frac{1}{2}}+3 x_{0}^{2} Q^{-1}\right] \quad(\text { for } Q \gg 1) .
\end{aligned}
$$

Overall, starting from a narrow PDF centered about $x=x_{0}$, the PDF undergoes three stages. The first is the Gaussian evolution ending with the broadening of the PDF around $t_{f} \sim\left(2 \mu x_{0}^{2}\right)^{-1}$, described by Eq. (60). This stage reflects the initial Brownian motion where the white noise is balanced by the inertia $d x / d t$, terminating when the effect of nonlinear force becomes non-negligible, leading to a slight broadening of the PDF. The nonlinear force shortly gives rise to a coherent force, initiating the second stage of yet another Gaussian evolution where fluctuation evolves with the effective frictional force as $\mu_{o} \propto \mu\langle x\rangle^{2}$ governed by the O-U process. With further increases in time, the PDF finally settles in to the final stationary quartic exponential PDF in Eq. (61) for $t_{f} \gg t_{c}$ where

$$
t_{c} \sim \sqrt{\frac{3}{4 D \mu}} .
$$

[See Appendix $\mathrm{H}$ for an alternative derivation of Eq. (62).] The critical time $t_{c}$ in Eq. (62) increases as $D$ decreases, similarly to the case of $x_{0}=0$ given in Eq. (35), demonstrating that the final relaxation time to a stationary PDF depends on $D$ and $\mu$ (and not on $x_{0}$ ). Aforementioned different stages of PDF evolution and the two characteristic transition times $\left[t_{f} \sim\right.$ $\left(2 \mu x_{0}^{2}\right)^{-1}$ and Eq. (62)] are confirmed from the exact solutions obtained by numerical calculations in Sec. 3 .

The odd terms in $r$ signify the asymmetry of the PDF around its peak, where the PDF on the left side of the peak is enhanced over that on the right side of the peak. Recalling that $x_{0}>0$ in our analysis, this illustrates the enhancement of the PDF around $x_{0}=0$ as the PDF moves towards $x_{f}=0$. This asymmetry property is also confirmed by numerical calculation in Sec. 3.

Finally, the peak amplitude of the PDFs is determined by the normalization $N$ in Eq. (15), which is determined by $\int d x_{f} p\left(x_{f}, t_{f} ; x_{0}, 0\right)=1$. For the Gaussian PDF of the form $p(x, t)=N e^{-\beta x^{2}}$, the peak amplitude $N=\sqrt{\beta / \pi} \propto \beta^{1 / 2}$ while the width is proportional to $\beta^{-1 / 2}$. Thus, for the Gaussian PDFs in Eqs. (61)-(62), the behavior of the peak amplitude of the PDFs can easily be inferred from the effective inverse temperature $\beta$. This is discussed in Sec. III together with the interpretation of the numerical results. For the quartic PDF of the form $p(x, t)=N e^{-\beta x^{4}}, N \propto \beta^{-1 / 4}$ (see Appendix A for its property).

\section{EXACT PDFS FROM THE FOKKER-PLANCK EQUATION}

To complement the analytic study in Sec. II, we compute numerical solutions of the time-dependent PDFs. While it would have been possible to obtain the marginal PDF $p(x, t)$ by stochastic simulation of Eq. (1) or (6), we instead solve the 

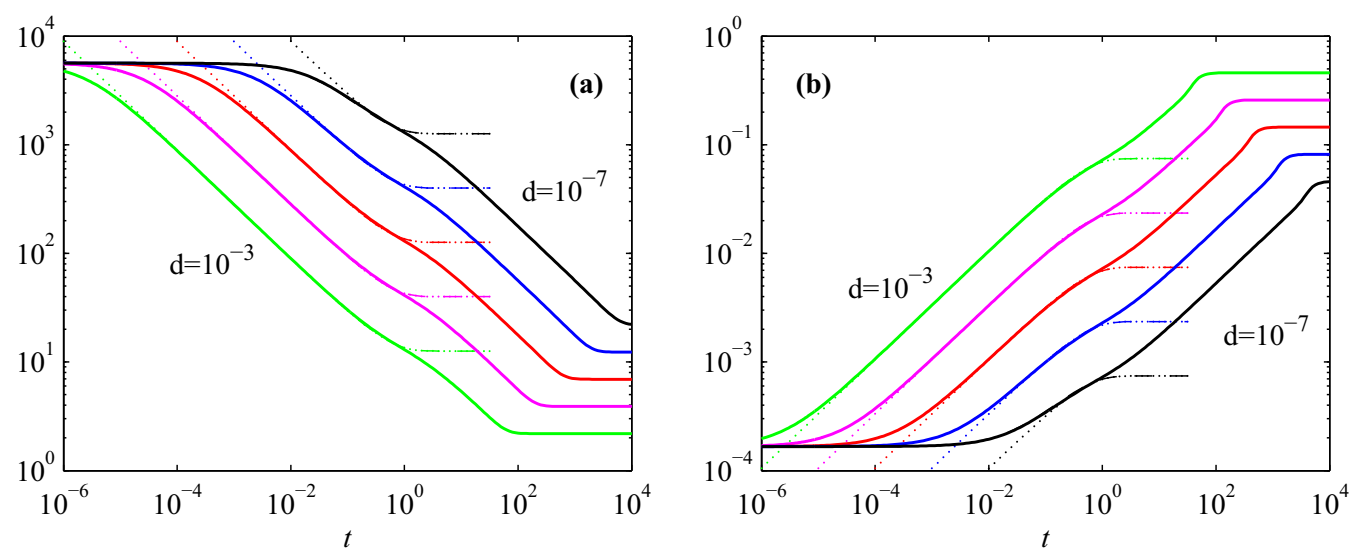

FIG. 2. (a) peak amplitudes; (b) widths at half-peak. The heavy solid lines are the cubic case with initial condition (64), the dash-dotted lines are the linear case with initial condition (64), and the dotted lines are the linear case with a $\delta$-function initial condition. The five different lines in each set correspond to $d=10^{-3}$ to $10^{-7}$, from left to right as indicated.

corresponding Fokker-Planck equations (e.g., Refs. [1,2]):

$$
\frac{\partial p}{\partial t}=d \frac{\partial^{2} p}{\partial x^{2}}+\frac{\partial}{\partial x}\left(x^{n} p\right)
$$

where $n=1$ for the linear O-U process, and $n=3$ for the cubic process. For convenience, we use $d=D / 2$ where $D$ is defined in Eq. (2). Without loss of generality, we also set $\mu_{0}=1$ for $n=1$ and $\mu=1$ for $n=3$ in Eq. (63). The entire equation can always be rescaled to make this coefficient one, which is convenient numerically to reduce the number of parameters in the problem.

To solve these equations numerically, we begin by restricting the interval in $x$ to $[-1,1]$, rather than the original $[-\infty, \infty]$. There is again no real loss in generality involved here; by suitably rescaling $x, t$, and/or $d$, any finite interval can always be mapped to $[-1,1]$. As long as $d$ and the initial condition are chosen such that $p$ would be negligible outside this interval anyway, then solving Eq. (63) only on $[-1,1]$, and with $p=0$ boundary conditions, should yield results in good agreement with the analytic formulations. The numerical solution involves second-order accurate finite differencing in $x$, using up to $M=4 \times 10^{6}$ grid points. The time stepping is also second-order accurate, with step sizes as small as $\Delta t=10^{-7}$. Both $M$ and $\Delta t$ were varied to ensure accuracy to within at least $0.1 \%$. Especially in the later stages of the evolution, once an initially narrow peak has started to broaden considerably, $M$ can also be decreased and $\Delta t$ increased, without loss of accuracy.

The initial condition was taken as

$$
p=\frac{1}{\sqrt{\pi 10^{-8}}} \exp \left[-\frac{(x-0.7)^{2}}{10^{-8}}\right]
$$

that is, a Gaussian with a peak at $x_{0}=0.7$ and a width $1.7 \times$ $10^{-4}$. The question then is how this initial condition evolves toward the final equilibrated state, either $p \propto \exp \left(-x^{2} / 2 d\right)$ for the linear process or $p \propto \exp \left(-x^{4} / 4 d\right)$ for the cubic process, with the constant of proportionality in each case fixed by $\int p d x=1$ (see Appendix A). For the linear process, an analytic solution for the entire time evolution given in Eq. (3) takes the following form:

$$
p(x, t)=\frac{1}{\sqrt{2 \pi d}} \frac{1}{\sqrt{1-\kappa e^{-2 t}}} \exp \left[-\frac{\left(x-x_{0} e^{-t}\right)^{2}}{2 d\left(1-\kappa e^{-2 t}\right)}\right],
$$

where $\kappa$ is an arbitrary constant. Taking $\kappa=1$ would correspond to a $\delta$-function initial condition, whereas $\kappa=$ $1-10^{-8} / 2 d$ corresponds to the actual initial condition (64).

Figure 2 shows how the peak amplitudes and widths at half-peak evolve in time, for the five values $d=10^{-3}$ to $10^{-7}$. The heavy solid lines show the cubic case that we are ultimately most interested in. The dash-dotted lines show the linear case with initial condition (64), and the dotted lines the linear case with a $\delta$ function initial condition [that is, the dash-dotted lines have $\kappa=1-10^{-8} / 2 d$ in Eq. (65) whereas the dashed lines have $\kappa=1$ ]. If we first compare the two different initial conditions in the linear case, we see that a $\delta$-function initial condition relatively quickly (on a time scale $\sim 10^{-8} / d$ ) becomes indistinguishable from the initial condition (64). This is encouraging, as it indicates that all the theoretical analysis developed for a $\delta$-function initial condition is still relevant even for (64). The other point to note about the linear cases is how the evolution is indeed completed once $t \approx 1$, independent of $d$. Different stages of PDF evolution with the two transition times above agree with the analytical prediction summarized in Sec. II C.

Turning next to the cubic cases, we see that up to $t \approx 0.1$ they follow the corresponding linear cases almost exactly. The detailed structure continues to be Gaussian in this regime (as indicated also by further diagnostics below). For $t \geqslant O(1)$ the peaks continue to decrease and the widths to increase, before eventually settling in to the final equilibrated solutions where the peaks scale as $d^{-1 / 4}$ and the widths as $d^{1 / 4}$. Note also how the time required to reach the final stationary PDF clearly scales as $d^{-1 / 2}$, in agreement with the analytic predictions in Sec. II C.

Turning next to where $p$ is located, Fig. 3 shows two different ways of measuring this, the mean value $\langle x\rangle=\int x p d x$, and the position of the maximum value of $p$, call it $x_{\text {peak }}$. Up to $t \approx 1$ both measures are essentially indistinguishable from the expected result $x_{0} / \sqrt{1+2 t x_{0}^{2}}$ for small fluctuations $\left\langle(\delta x)^{2}\right\rangle \ll\langle x\rangle^{2}$; as fluctuations increase with time, deviations 

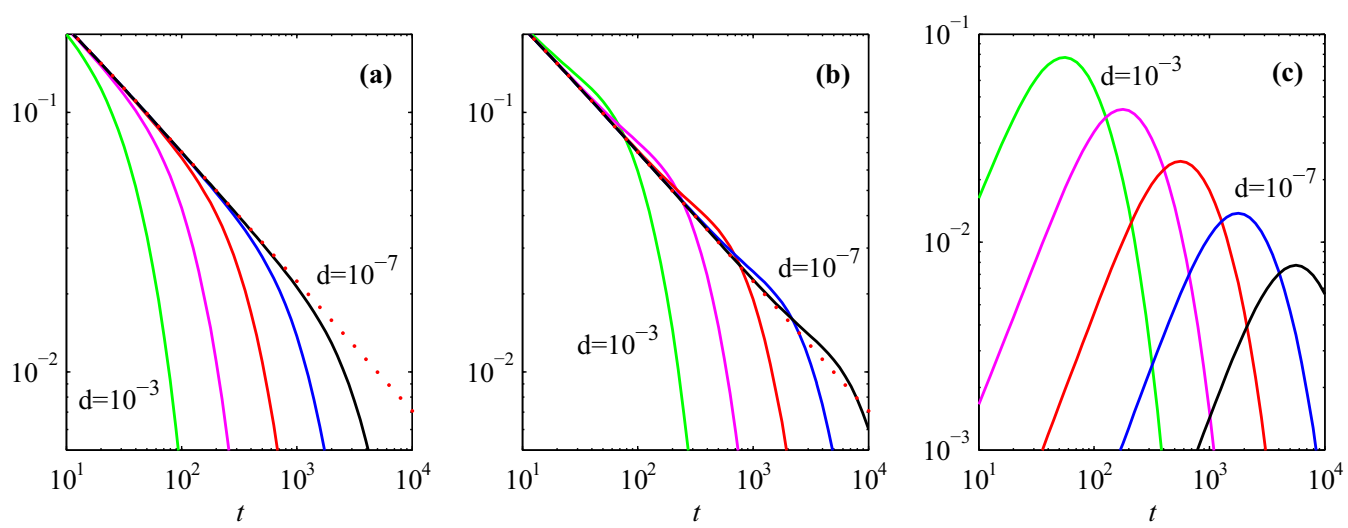

FIG. 3. (a) The mean value $\langle x\rangle$, for $d=10^{-3}$ to $10^{-7}$ as indicated. (b) $x_{\text {peak }}$, the location where $p$ takes its maximum value. In both panels the dotted line denotes $x_{0} / \sqrt{1+2 t x_{0}^{2}}$. (c) The difference, $x_{\text {peak }}-\langle x\rangle$.

do begin to appear as shown in the range $t>O(1)$ in Fig. 3. As predicted in Eq. (9), $\langle x\rangle$ tends to zero somewhat faster than expected from $x_{0} / \sqrt{1+2 t x_{0}^{2}}$, especially for the larger values of $d$. This is due to the contribution from fluctuations $\left\langle(\delta x)^{2}\right\rangle$ in Eq. (9), which increases with $d$. Since larger $d$ corresponds to greater variance, $\langle x\rangle$ tends to zero faster. That is, fluctuations lead to the enhanced dissipation of the mean value. Considering $x_{\text {peak }}$ next, this ultimately follows the same trend of tending to zero faster and with the same variation with $d$. It is interesting to note that for brief intermediate times these curves are slightly above $x_{0} / \sqrt{1+2 t x_{0}^{2}}$. The final panel in Fig. 3 shows the difference between these two measures of position. For all five values of $d$ there are times where this difference is surprisingly large, comparable to the larger of the two at the corresponding time.

The fact that these two measures of location give somewhat different answers is already indicative of the result noted above, that the PDF is expected to be asymmetric about its peak. This can be further quantified by computing the skewness $\int\left[(x-\langle x\rangle)^{2} / \sigma\right]^{3} p d x$, where $\sigma=\left[\int(x-\langle x\rangle)^{2} p d x\right]^{1 / 2}$ is the variance. Another interesting quantity is the kurtosis $\int\left[(x-\langle x\rangle)^{2} / \sigma\right]^{4} p d x$. Analytically one finds easily enough that a Gaussian profile has kurtosis 3, whereas the final quartic profile has kurtosis 2.19 (e.g., see Appendix A). A third quantity to consider is the ratio of the variance $\sigma$ to the half-peak width. For this one finds analytically that a Gaussian has 0.425 , whereas a quartic has 0.319. Figure 4 shows how these three diagnostics evolve in time. The skewness starts and ends at zero, as expected, but at intermediate times reaches a peak negative value of -0.56 , reflecting this difference in the two location measures in Fig. 3. This negative value of skewness is predicted in Sec. II C. The kurtosis similarly starts at 3 and ends at 2.19, as expected, but at intermediate times actually increases to a peak of 3.37. The variance-to-width ratio follows the same pattern as the kurtosis.

These results are interesting in the following two aspects. First, this clearly shows that the stationary PDF is very different from the nonequilibrium PDF. Second, the broadening of the $\mathrm{PDF}$ in the intermediate time before reaching the stationary PDF is reminiscent of a cyclic geodesic solution in Ref. [18], suggesting an important role of nonlinear interaction (force) in a geodesic. Detailed discussion on the implications of these results for information change is provided in Ref. [35]. The last point to note about all three diagnostics is how the curves for different values of $d$ are essentially identical, but offset in time according to a $d^{-1 / 2}$ scaling. This is another reflection of the result derived analytically and discussed in Sec. II C
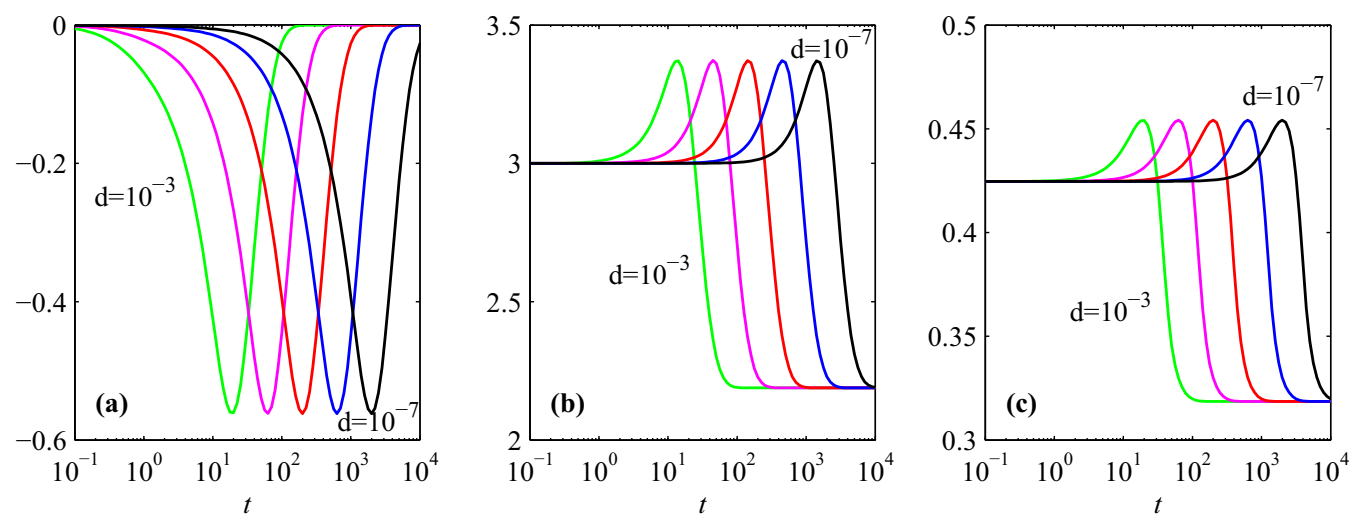

FIG. 4. (a) The skewness $\int\left[(x-\langle x\rangle)^{2} / \sigma\right]^{3} p d x$, (b) the kurtosis $\int\left[(x-\langle x\rangle)^{2} / \sigma\right]^{4} p d x$, and (c) the ratio of variance to half-peak width. In each panel $d$ varies from left to right as $10^{-3}$ to $10^{-7}$. Note the $\propto d^{-1 / 2}$ scaling in time. 
[see. Eq. (62)] and also seen in Fig. 2 that the final adjustment time scale for the cubic process scales as $d^{-1 / 2}$.

\section{CONCLUSION}

We have presented time-dependent PDFs in a cubic nonlinear stochastic process where the frictional force is given by a cubic nonlinearity. Analytically, we applied an instanton method based on a path integral formulation to a nonlinear system in the limit of weak noise (small $D$ ) and proposed a new nonlinear time transformation to solve nonlinear instanton (saddle-point) equations. We predicted a PDF which in general involves an integral and elucidated the effect of nonlinear interaction on enhanced dissipation in relaxation processes. Useful local time-dependent PDFs were presented in certain limits (e.g., in the short and long time limits). In particular, a transient PDF in the cubic process was shown to be asymmetric around its peak while the relaxation time $t_{f}>t_{c} \sim \frac{1}{\sqrt{\mu D}}$ in Eq. (62) depends on $D$, increasing as $D$ decreases. This sharply contrasts a linear stochastic process where transient PDFs are Gaussian and symmetric while the relaxation time $t_{f}$ to the final stationary PDF is independent of the diffusion coefficient $D$. The $D$ dependence of the relaxation time for a cubic process reflects a close interlinking between space and time in nonlinear relaxation processes. Alternatively, time flows at a different rate depending on the coordinate. We also demonstrated the utility of generalized two-family trigonometric functions in solving nonlinear equations. Numerical simulation of the Fokker-Planck equation revealed detailed evolution of the time-dependent PDF; analytical and numerical results agreed on overall PDF evolution, in particular, transition times for different evolutions (e.g. relaxation time $t_{f}$ ) and asymmetry, as noted in Sec. II C and 3. Furthermore, it highlighted that transient PDFs behave drastically differently from the stationary PDFs in regard to the asymmetry (skewness) and kurtosis. Of particular interesting is the settling in to a symmetric and narrow stationary PDF only after undergoing a transient state with asymmetric and broad PDF.

The generality of our methodology and predicted exponential PDF are reminiscent of the possibility of transforming any automonous nonlinear Langevin equation driven by a white noise to the Brownian motion, while our proposed nonlinear time transformation plays a role of random time change: the so-called Lamperti transformation [2,3]. The latter transforms away a nonlinear diffusion coefficient $(D)$ to a constant diffusion (e.g., see Refs. [36,37] and Theorems 7.37 and 7.39 and Remark 7.4, chap. 7 in Ref. [2]). Together with the change of variables, or change of measure (Girsanov transformation) which removes the drift term (i.e., $\frac{\partial V}{\partial x}$ in our case), the solution to any stochastic equation with time-independent coefficient can be obtained by the Brownian motion (e.g., see Refs. [2,3]). However, since the resulting Brownian motion depends on random time, it is not clear how to calculate transient PDFs by using this method. In comparison, our nonlinear time transformation seems to offer a systematic way of computing the PDFs in different limits. This opens a large scope for future study including the application of our method to other nonlinear stochastic processes. Of particular interest would be the inclusion of a linear (negative) force in the cubic process to investigate the dynamics of growth, phase transition, and long-term memory. A change of variables would then permit us to examine the Feller-branching process with a logistic growth (e.g., see Ref. [36]). Furthermore, the investigation of the change in information in nonlinear processes in terms of information length [17] is addressed in the accompanying paper [35].

\section{APPENDIX A: PROPERTY OF $p(x)=N \exp \left(-\beta x^{4}\right)$}

We first show how to fix $N$ by the unity of the total probability $\int_{-\infty}^{\infty} d x p(x)=1$ :

$$
\begin{aligned}
N^{-1} & =\int_{-\infty}^{\infty} d x e^{-\beta x^{4}} \\
& =2 \int_{0}^{\infty} d x e^{-\beta x^{4}} \\
& =\frac{1}{2} \beta^{-\frac{1}{4}} \int_{0}^{\infty} d y y^{-\frac{3}{4}} e^{-y} \\
& =\frac{1}{2} \beta^{-\frac{1}{4}} \Gamma\left(\frac{1}{4}\right),
\end{aligned}
$$

where the change of the variable $y=\beta x^{4}\left(d x=\frac{1}{4} \beta^{-\frac{1}{4}} y^{-\frac{3}{4}} d y\right)$ was used and $\Gamma(z)=\int_{0}^{\infty} d y y^{z-1} e^{-y}$ is the Gamma function. That is,

$$
p(x)=\frac{2 \beta^{\frac{1}{4}}}{\Gamma\left(\frac{1}{4}\right)} e^{-\beta x^{4}} .
$$

By using Eq. (A1), we can calculate the second and fourth moments as follows:

$$
\begin{aligned}
\left\langle x^{2}\right\rangle & =\int_{-\infty}^{\infty} d x x^{2} p(x)=2 N \int_{0}^{\infty} d x x^{2} e^{-\beta x^{4}} \\
& =\frac{N}{2} \beta^{-\frac{1}{4}} \beta^{-\frac{1}{2}} \int_{0}^{\infty} d y y^{-\frac{1}{4}} e^{-y} \\
& =\frac{\Gamma\left(\frac{3}{4}\right)}{\Gamma\left(\frac{1}{4}\right)} \beta^{-\frac{1}{2}}
\end{aligned}
$$

and

$$
\begin{aligned}
\left\langle x^{4}\right\rangle & =\int_{-\infty}^{\infty} d x x^{4} p(x)=2 N \int_{0}^{\infty} d x x^{4} e^{-\beta x^{4}} \\
& =\frac{N}{2} \beta^{-\frac{1}{4}} \beta^{-1} \int_{0}^{\infty} d y y^{\frac{1}{4}} e^{-y} \\
& =\frac{\Gamma\left(\frac{5}{4}\right)}{\Gamma\left(\frac{1}{4}\right)} \beta^{-1}=\frac{1}{4} \beta^{-1},
\end{aligned}
$$

where $\Gamma(z)=(z-1) \Gamma(z)$ was used for $z=\frac{5}{4}$ in the last line.

From Eq. (A2) and Eq. (A3), we find the kurtosis $\kappa$ :

$$
\kappa=\frac{\left\langle x^{4}\right\rangle}{\left\langle x^{2}\right\rangle^{2}}=\frac{1}{4}\left[\frac{\Gamma\left(\frac{1}{4}\right)}{\Gamma\left(\frac{3}{4}\right)}\right]^{2}=2.1884 .
$$

Thus, the quartic exponential PDF has kurtosis less than 3, indicating narrow width and flatness. Note that the Gaussian PDF has $\kappa=3$. 
APPENDIX B: PATH INTEGRAL IN EQ. (12)

For Gaussian statistics with vanishing first moment, the prescription of the second moment given by Eq. (2) is sufficient. It is simply because all odd moments vanish while even moments can be expressed as a product of second moments. Note that even if the forcing is Gaussian, statistics of $x$ can be non-Gaussian because of the nonlinearity of the dynamical equation. An equivalent way of prescribing the second moment (2) for the Gaussian forcing is to introduce the probability density function for $\xi$ as follows [12,26,33]:

$$
d[\rho(\xi)]=\mathcal{D} \xi \exp \left\{-\frac{1}{2} \int d t D^{-1} \xi(t)^{2}\right\} .
$$

This is a Gaussian distribution for $\xi(t)$. The average value of a quantity $Q$ is then computed as

$$
\langle Q\rangle=\int d[\rho(\xi)] Q .
$$

When the average value of a functional of $x$ (i.e., $\langle Q[x]\rangle)$ is required, the constraint should be imposed that $\xi$ and $x$ satisfy the original equation (1). This can be done by inserting an identity with a $\delta$ function, which enforces Eq. (1), as

$$
\begin{aligned}
1 & =\int \mathcal{D} x \delta\left[\frac{d x}{d t}+\frac{\partial V}{\partial x}-\xi\right] J \\
& \propto \int \mathcal{D} x \mathcal{D} \bar{x} \exp \left\{i \int d t \bar{x}\left[\frac{d x}{d t}+\frac{\partial V}{\partial x}-\xi\right]\right\} J
\end{aligned}
$$

where $J=J\left[\frac{\partial \xi}{\partial x}\right]$ is the Jacobian due to the change of variables for the delta function. Let us show in detail how this is done. Starting from the definition,

$$
\begin{aligned}
\langle Q[x]\rangle= & \int \mathcal{D} \xi Q[x] \exp \left\{-\frac{1}{2 D} \int d t \xi(t)^{2}\right\} \\
= & \int \mathcal{D} \xi \mathcal{D} x Q[x] \delta\left[\frac{d x}{d t}+\frac{\partial V}{\partial x}-\xi\right] J \\
& \times \exp \left\{-\frac{1}{2 D} \int d t \xi(t)^{2}\right\} \\
= & \int \mathcal{D} \xi \mathcal{D} x \mathcal{D} \bar{x} Q[x] \exp \left\{i \int d t \overline { x } \left[\frac{d x}{d t}\right.\right. \\
& \left.\left.+\frac{\partial V}{\partial x}-\xi\right]\right\} J \exp \left\{-\frac{1}{2 D} \int d t \xi(t)^{2}\right\} \\
= & \int \mathcal{D} x \mathcal{D} \bar{x} Q[x] e^{-S},
\end{aligned}
$$

leading to $S$ given in Eq. (12). In Eq. (B3), Eq. (B2) was used to obtain the third line; $J=e^{-\psi}$ and $\psi=-\frac{3}{2} \mu x^{2}$ [see, e.g., Eq. (96)-(97) in Ref. [33], Eq. (2.10) in Ref. [12]] for $V(x)=\mu x^{4} / 4$ and the Gaussian integral over $\xi$ were used to obtain the last line. Taking $Q[x]=\delta\left(x\left(t_{f}\right)=x_{f}\right) \delta[x(0)=$ $\left.x_{0}\right]$ gives us Eq. (11). Note that $\bar{x}$ is a conjugate variable, which acts as a mediator between the forcing $\xi$ and dynamical variable $x$.

\section{APPENDIX C: NONLINEAR TRANSFORMATION [EQ. (18)]}

Let us consider a homogeneous cubic equation $\frac{d x}{d t}=-\mu x^{3}$. The usual way of solving this equation is to separate variables and integrate to obtain

$$
x(t)=\frac{x_{0}}{\sqrt{1+2 \mu t x_{0}^{2}}},
$$

where the initial condition $x(t=0)=x_{0}$ is used. To elucidate how the nonlinear transformation defined in Eq. (18) works, we rewrite $\frac{d x}{d t}=-\mu x^{3}$ as follows:

$$
0=\frac{d x}{d t}+\mu x^{3}=x^{2}\left(\frac{d x}{d \tau}+\mu x\right) .
$$

The solution to Eq. (C2) is $x(\tau)=x_{0} e^{-\mu \tau}$ where $x_{0}=x(\tau=$ $0)=x(t=0)$. To obtain $x(t)$, we use $x\left(\tau_{1}\right)=x_{0} e^{-\mu \tau_{1}}$ in Eq. (25):

$$
\begin{aligned}
t & =\int_{0}^{\tau} d \tau_{1} \frac{1}{\left[x\left(\tau_{1}\right)\right]^{2}}=\frac{1}{2 \mu x_{0}^{2}}\left(e^{2 \mu \tau}-1\right) \\
& =\frac{1}{2 \mu x_{0}^{2}}\left\{\frac{x_{0}^{2}}{[x(t)]^{2}}-1\right\} .
\end{aligned}
$$

Solving Eq. (C3) for $x(t)$ gives the same solution [Eq. (C1)].

\section{APPENDIX D: GENERALIZED TWO-FAMILY TRIGONOMETRY FUNCTIONS}

The generalized sine function with two parameters $p, q$, where $p>1$ and $q>1$, is defined through its inverse function [34]

$$
\arcsin _{p, q}(x)=\int_{0}^{x} d t\left(1-t^{q}\right)^{-1 / p},
$$

where $x=[0,1]$. Note that when $p=q=2$, Eq. (D1) recovers the definition of the usual $\arcsin (x)$. When $x=1$, Eq. (D1) defines the generalized $\pi_{p, q}$ as

$$
\arcsin _{p, q}(1)=\int_{0}^{1} d t\left(1-t^{q}\right)^{-1 / p}=\frac{\pi_{p, q}}{2},
$$

which again recovers $\pi / 2$ when $p=q=2$. We note that $\sin _{p, q}(x)$ is a monotonically increasing function of $x$, mapping $[0,1] \rightarrow\left[0, \pi_{p, q} / 2\right]$, and Eq. (D1) can also be written in terms of Gaussian hypergeometric function.

The $(p, q)$-cosine is defined as

$$
\cos _{p, q}(x)=\frac{d \sin _{p, q}(x)}{d x}=\left\{1-\left[\sin _{p, q}(x)\right]^{q}\right\}^{1 / p}
$$

where $x$ is a real number. Hence, $\cos _{p, q}(x)$ is strictly decreasing on $\left[0, \pi_{p, q} / 2\right], \cos _{p, q}(0)=1, \cos _{p, q}\left(\pi_{p, q} / 2\right)=0$ and satisfies the following identity:

$$
\left|\sin _{p, q}(x)\right|^{q}+\left|\cos _{p, q}(x)\right|^{p}=1,
$$

which is Eq. (29) with $x=z_{f}$. 


\section{APPENDIX E: DERIVATION OF EQ. (32)}

To show the identity Eq. (32), we let the left-hand side of Eq. (32) be $I_{1}$ and reexpress it as follows:

$$
\begin{aligned}
I_{1} & \equiv \int_{0}^{z} d z_{1} \frac{1}{\left(1-z_{1}^{3}\right)^{5 / 3}} \\
& =\int_{0}^{z} d z_{1}\left[\frac{1-z_{1}^{3}}{\left(1-z_{1}^{3}\right)^{5 / 3}}+\frac{z_{1}^{3}}{\left(1-z_{1}^{3}\right)^{5 / 3}}\right] \\
& \equiv I_{0}+\int_{0}^{z} d\left[\left(1-z_{1}^{3}\right)^{-2 / 3}\right] \frac{z_{1}}{2} \\
& =I_{0}+\frac{1}{2} \frac{z}{\left(1-z^{3}\right)^{2 / 3}}-\frac{1}{2} \int_{0}^{z} d z_{1} \frac{1}{\left(1-z_{1}^{3}\right)^{2 / 3}} \\
& =I_{0}+\frac{1}{2} \frac{z}{\left(1-z^{3}\right)^{2 / 3}}-\frac{1}{2} I_{0}=\frac{1}{2} I_{0}+\frac{1}{2} \frac{z}{\left(1-z^{3}\right)^{2 / 3}} \\
& =\frac{1}{2}\left[\int_{0}^{z} d z_{1}\left(1-z_{1}^{3}\right)^{-2 / 3}+z\left(1-z^{3}\right)^{-2 / 3}\right],
\end{aligned}
$$

obtaining Eq. (32) in the text. Here $I_{0}$ in Eqs. (E3)-(E5) is defined as

$$
I_{0} \equiv \int_{0}^{z} d z_{1} \frac{1}{\left(1-z_{1}^{3}\right)^{2 / 3}},
$$

and integration by parts is used to obtain Eq. (E4) from Eq. (E3).

\section{APPENDIX F: FOR $y-\gamma>0$}

In this case, we obtain from Eq. (38) and Eq. (25)

$$
t=\frac{1}{6 \mu B^{\frac{2}{3}}} I_{0},
$$

where

$$
I_{0}=\int_{1}^{y_{f}} \frac{d y}{y^{\frac{2}{3}}} \frac{1}{(y-\gamma)^{\frac{2}{3}}} .
$$

Here $y=e^{6 \mu t}$ and $y_{f}=e^{6 \mu \tau_{f}}$. Similarly, Eq. (38) can be written as

$$
\begin{gathered}
S_{\text {eff }}=\frac{\mu B^{\frac{4}{3}}}{3 D} I_{1}=\frac{\mu}{6 D}\left\{6 \mu t_{f}|\alpha B|+3 B^{\frac{4}{3}}\left[y^{1 / 3}(y-\gamma)^{\frac{1}{3}}\right]_{1}^{y_{f}}\right\} \\
I_{1}=\int_{1}^{y_{f}} d y \frac{y^{\frac{1}{3}}}{(y-\gamma)^{\frac{2}{3}}} .
\end{gathered}
$$

In the second line in Eq. (F3), we used Eq. (F1) and the following identity (similar to that used in Appendix C):

$$
I_{1}=\frac{1}{2}\left\{\gamma I_{0}+3\left[y^{1 / 3}(y-\gamma)^{\frac{1}{3}}\right]_{1}^{y_{f}}\right\} .
$$

\section{APPENDIX G: DERIVATION OF EQ. (45)}

We rewrite $I_{0}$ in Eq. (42) in terms of $I_{1}$ in Eq. (44) as

$$
\begin{aligned}
I_{0} & =\int_{1}^{y_{f}} d y \frac{y^{-\frac{2}{3}}}{(\gamma-y)^{\frac{2}{3}}} \\
& =\frac{1}{\gamma}\left[I_{1}+J\right],
\end{aligned}
$$

where

$$
\begin{aligned}
J & =\int_{1}^{y_{f}} d y y^{-\frac{2}{3}}(\gamma-y)^{\frac{1}{3}} \\
& =3\left\{\left[y^{\frac{1}{3}}(\gamma-y)^{\frac{1}{3}}\right]_{1}^{y_{f}}+\frac{1}{3} \int_{1}^{y_{f}} d y y^{\frac{1}{3}}(\gamma-y)^{-\frac{2}{3}}\right\} \\
& =3\left[y^{\frac{1}{3}}(\gamma-y)^{\frac{1}{3}}\right]_{1}^{y_{f}}+I_{1} .
\end{aligned}
$$

By using Eq. (G2) in Eq. (C1), we obtain

$$
\gamma I_{0}=2 I_{1}+3\left[y^{\frac{1}{3}}(y-\gamma)^{\frac{1}{3}}\right]_{1}^{y_{f}},
$$

which gives Eq. (45).

\section{APPENDIX H: ALTERNATIVE DERIVATION OF THE RELAXATION TIME TO THE STATIONARY PDF}

To estimate the relaxation time to the stationary PDF, we let $r=r^{(0)}+r^{(1)}$ in Eq. (61) by assuming a small $Q^{-1}$ and find

$$
r=\left(\frac{2 D}{\mu}\right)^{\frac{1}{4}}-\frac{3}{4 \sqrt{2 \mu t_{f}}} .
$$

By comparing the two terms in $r$ above, we conclude that the critical time $t_{c}$ required to relax into the equilibrium PDF satisfies

$$
t_{f}>\frac{9}{32 \sqrt{2 D \mu}}\left(\equiv t_{c}\right) .
$$

[1] H. Risken, The Fokker-Planck Equation: Methods of Solution and Applications: Methods of Solutions and Applications, 3rd ed. (Springer, New York, 2013).

[2] F. Klebaner, Introduction to Stochastic Calculus with Applications (Imperial College Press, London, 2012), ch. 5.5 .

[3] C. Gardiner, Stochastic Methods, 4th ed. (Springer, New York, 2008), ch. 4.4.
[4] H. Haken, Information and Self-organization: A Macroscopic Approach to Complex Systems, 3rd ed. (Springer, New York, 2006), pp. 63-64.

[5] B. Rényi, Proceedings of the Fourth Berkeley Symposium on Mathematical Statistics and Probability, edited by J. Neyman (University of California Press, Berkeley, 1961), p. 547.

[6] A. D. Wissner-Gross and C. E. Freer, Phys. Rev. Lett. 110, 168702 (2013). 
[7] A. Caticha, AIP Conf. Proc. 617, 302 (2001).

[8] D. Acosta, P. F. de Córdoba, J. M. Isidro, and J. L. G. Santander, arXiv:1107.1898 (2011).

[9] S.-I. Itoh, K. Itoh, M. Yagi, M. Kawasaki, and A. Kitazawa, Phys. Plasmas 9, 1947 (2002).

[10] C. Tsallis, Introduction to Nonextensive Statistical Mechanics: Approaching a Complex World (Springer, New York, 2009).

[11] S. P. Das and G. F. Mazenko, J. Stat. Phys. 149, 643 (2012).

[12] P. Hänggi, Z. Phys. B 75, 275 (1989).

[13] J. Anderson and E. Kim, Plasma Phys. Controlled Fusion 52, 012001 (2010).

[14] E. Kim, H.-L. Liu, and J. Anderson, Phys. Plasmas 16, 052304 (2009).

[15] E. Kim and P. H. Diamond, Phys. Rev. Lett. 88, 225002 (2002).

[16] E. Kim and P. H. Diamond, Phys. Plasmas 9, 71 (2002).

[17] J. Heseltine and E. Kim, J. Phys. A: Theor. Math. 49, 175002 (2016).

[18] E. Kim, U. J. Lee, J. Heseltine, and R. Hollerbach, Phys. Rev. E 93, 062127 (2016).

[19] S. B. Nicholson and E. Kim, Phys. Lett. A 379, 83 (2015).

[20] A. P. L. Newton, E. Kim, and H.-L. Liu, Phys. Plasmas 20, 092306 (2013).

[21] E. Kim and S. Nicholson, Phys. Lett. A 379, 1613 (2015).

[22] R. Hermann, Fractional Calculus: An Introduction for Physicists (World Scientific, Singapore, 2011).

[23] J. Anderson, E. Kim, and S. Moradi, Phys. Plasmas 21, 122109 (2014).

[24] M. H. Pinsonneault, S. D. Kawaler, S. Sofia, and P. Demarque, Astrophys. J. 338, 424 (1989).
[25] E. T. Lu, Phys. Rev. Lett. 74, 2511 (1995).

[26] J. Zinn-Justin, Quantum Field Theory and Critical Phenomena (Oxford Science Publications, Oxford, 2008), Chs. 2-4.

[27] J. Zinn-Justin and U. D. Jentschura, Ann. Phys. 313, 197 (2004); 313, 269 (2004).

[28] U. D. Jentschura and J. Zinn-Justin, Ann. Phys. 326, 2186 (2011).

[29] U. D. Jentschura, A. Surzhykov, and J. Zinn-Justin, Ann. Phys. 325, 1135 (2010).

[30] R. P Feynman and A. R. Hibbs, Quantum Mechanics and Path Integrals (McGraw-Hill, New York, 1965), Ch. 2; R. P. Feynman, Statistical Mechanics (W. A. Benjamin, New York, 1972), Ch. 3.

[31] G. 't Hooft, Phys. Rev. Lett. 37, 8 (1976).

[32] V. Gurarie and A. Migdal, Phys. Rev. E 54, 4908 (1996); G. Falkovich, I. Kolokolov, V. Lebedev, and A. Migdal, ibid. 54, 4896 (1996).

[33] U. Seifert, in Soft Matter, From Synthetic to Biological Materials, Lecture Notes of the 39th IFF Spring School 2008, edited by J. K. G. Dhont, G. Gompper, G. Nägele, D. Richter, and R. G. Winkler (Research Center Jülich, Jülich, 2008), pp. $1-30$.

[34] D. E. Edmunds, P. Gurka, and J. Lang, J. Math. Anal. Appl. 420, 1680 (2014); L. Boulton and G. J. Lord, Proc. R. Soc. London A 471, 20140642 (2015); B. A. Bhayo and M. Vuorinen, J. Approx. Theory 164, 1415 (2012).

[35] E. Kim and R. Hollerbach (unpublished).

[36] A. Lambert, Ann. Appl. Prob. 15, 1506 (2005).

[37] L. E. Dubins and G. Schwarz, Proc. Nat. Acad. Sci. USA 53, 913 (1965). 